\title{
RESEARCH AND APPLICATION OF GIS DATA UPDATE TECHNOLOGY
}

\author{
JanPing Pan, QingLing Xu, ChunHua Yang \\ Chongqing Institute of Surveying and Mapping, NASMG, Chongqing, China - pjplj@126.com
}

Keywords: GIS Data; Data Update; Raster; Vector

\begin{abstract}
:
This paper summed up 3 kinds of GIS data update ways started from the actual production, as these data are raster to raster data, raster to vector data and vector to vector data. We described the most advanced and most effective ways to update each kind of data, solved lots of insufficient exist in current GIS data update ways effectively, such as long update time, low update efficiency, data redundancy and can't query and analysis based on time. From the actual production project, those ways we described were proven to be effective and feasible.
\end{abstract}

\section{INTRODUCTION}

As a unified framework for spatial orientation and basic data for spatial analysis, GIS data is a kind of basic and strategic resource which involved in national security and economic development. Due to the rapid development of national construction, the related GIS data is changed in moment, and the existing geographic information can not reflect the latest status, improve the update frequency and accuracy become very important.

Until now, there are many deficiencies exist in GIS data update, such as long update time, low update efficiency and data redundancy, etc. To update the GIS data completely and timely to meet the needs of variety applications is the focus of this article. We summed and improved the methods to update all 3 kinds of data, and summed up the feasible methods which can improve productivity based on actual production.

\section{TYPE OF UPDATED GEOGRAPHIC INFORMATION}

Analysis from the change status of geographic information, changes including newly increased geographic information elements, and disappeared of original elements, information still exists, but the existence of the state has changed. Analysis from the amount of geographic information, the amount of updated geographic information has a great relationship between elements category, economic development status, location and time interval. Different elements have different update amount, they bound up human activities closely, such as construction, as the rapid development of road and railway network brought a lot of changes in the traffic information. Analysis from the data resouces, geographic information update including raster to raster data updates, raster to vector data updates and vector to vector data updates.

\section{RESEARCH AND APPLICATION ON UPDATE TECHNOLOGY}

\section{Raster to raster data update}

Remote sensing image information obtained by different remote sensors have different information superiority. To produce a new image data by data fusion technology with advantage of information can reduce or inhibit the ambiguity, incompleteness, uncertainty and error, and can maximize the use of various information. For example, to fuse the multispectral and panchromatic band image data from Landsat, spatial resolution of the result image can be increased from $30 \mathrm{~m}$ to $15 \mathrm{~m}$, and can meet needs of basic GIS data update of 1:25000 and 1:50000. However, the $15 \mathrm{~m}$ spatial resolution can not meet the requirements for larger-scale basic GIS data update obviously. To fuse the aerial imagery (1:3500 scale and $0.6 \mathrm{~m}$ spatial resolution) and 
multispectral band of Landsat can effectively improve the spatial resolution of the image in order to obtain higher quality information.

In a general way, to realize raster data update will use spatial adjustment and data fusion method.

$$
\begin{aligned}
& x_{0}=\left(\sum_{i=0}^{t}\right)\left(\sum_{j=0}^{t}\right) a_{k} x^{i-j} y^{j} \\
& y_{0}=\left(\sum_{i=0}^{t}\right)\left(\sum_{j=0}^{t}\right) b_{k} x^{i-j} y^{j}
\end{aligned}
$$

Where $t_{\text {is }}$ Polynomial order, $a_{k}, b_{k}$ is coefficient, $k=\frac{i^{*} i+\mathrm{j}}{2}+j, t$ take 2 always can meet the requirements in adjustment processing. For aerial images, the strict colinearity equation is more used in ortho-rectification, the colinearity equation as follow:

$x=-f \frac{a_{1}\left(X-X_{s}\right)+b_{1}\left(Y-Y_{s}\right)+c_{1}\left(\mathrm{Z}-\mathrm{Z}_{\mathrm{s}}\right)}{a_{3}\left(\mathrm{X}-\mathrm{X}_{\mathrm{s}}\right)+\mathrm{b}_{3}\left(\mathrm{Y}-\mathrm{Y}_{\mathrm{s}}\right)+c_{3}\left(Z-Z_{s}\right)}$
$y=-f \frac{a_{2}\left(X-X_{s}\right)+b_{2}\left(Y-Y_{s}\right)+c_{2}\left(\mathrm{Z}-\mathrm{Z}_{\mathrm{s}}\right)}{a_{3}\left(\mathrm{X}-\mathrm{X}_{\mathrm{s}}\right)+\mathrm{b}_{3}\left(\mathrm{Y}-\mathrm{Y}_{\mathrm{s}}\right)+c_{3}\left(Z-Z_{s}\right)}$

Where $x, y$ is coordinate of image point, $f$ is focal length, $a_{i}, b_{i}, c_{i}(i=1,2,3)$ is the direction cosine, $\mathrm{X}, \mathrm{Y}, \mathrm{Z}$ is coordinate of ground point, $\mathrm{X}_{\mathrm{s}}, \mathrm{Y}_{\mathrm{s}}, \mathrm{Z}_{\mathrm{s}}$ is coordinate in the auxiliary coordinate system of ground point. Those area be changed deeply have to use digital elevation model to rectify, those area with little change can input a constant elevation to rectify.

Principal component instead and HIS transformation are the ways always be used in data fuse. Principal component instead method calculate the principal component of low-resolution image firstly, and then make histogram matching to high-
"Polynomial transformation" is a method more used in spatial adjustment for multi-spectral image, the change matrix as follows:

resolution image, and then use new high-resolution image generate from histogram matching result to instead the principal component of low-resolution image. Technical flowchart as Figure 1-1:

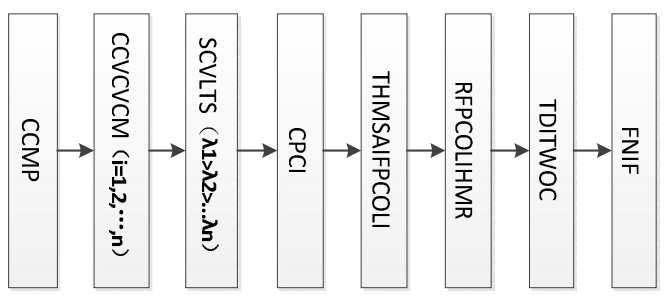

Figure 1-1 Technical flowchart of principle component instead

CCMP: Calculate correlation matrix produced by image.

CCVCVCM: Calculate characteristic value and characteristic vector based on correlation matrix.

SCVLTS: Sort the characteristic value from large to small.

CPCI: Calculate principal component image.

THMSAIFPCOLI: To do histogram matching between spatial adjusted image and the first principal component of the original low-resolution image.

RFPCOLIHMR: Replace the first principal component of the original low-resolution image by the histogram matching result.

TDITWOC: To do inverse transformation with other component

FNIF: Form new image by fusion.

HIS transformation method is forward transformation of IHS after give RGB values to the 3 bands of low-resolution image, and take the place of lightness by gray value of high-resolution image, then conduct IHS inverse transformation to get new RGB components which is color image formation, and then assigned low-resolution images to form color composite image, and then extract 3 bands in 
low resolution. HIS forward transformation formula is as follows:

$$
\begin{aligned}
& {\left[\begin{array}{l}
I \\
v_{1} \\
v_{2}
\end{array}\right]=\left[\begin{array}{l}
\frac{1}{\sqrt{3}} \frac{1}{\sqrt{3}} \frac{1}{\sqrt{3}} \\
\frac{1}{\sqrt{6}} \frac{1}{\sqrt{6}}-\frac{2}{\sqrt{6}} \\
\frac{1}{\sqrt{2}}-\frac{1}{\sqrt{2}} 0
\end{array}\right]\left[\begin{array}{l}
R \\
G \\
B
\end{array}\right]} \\
& H=\operatorname{arctg}\left(\frac{v_{2}}{v_{1}}\right) \\
& \mathrm{S}=\sqrt{v_{1}^{2}+v_{2}^{2}}
\end{aligned}
$$

Where $I$ is intension, $H$ is hue, $\mathrm{S}$ is saturation.

We can get image data with the same resolution and difference time phase through the above transformations, and then subtract spectral feature vector of each pixel in the two time phase image by change vector analysis to get multiple dimension difference image, and then conduct threshold segmentation to those changes in the spectral change vector set, as result, we get a seed training set, and get change detection result based on the similarity calculation.

\section{Vector to raster data update}

Changes monitoring related technique is the main method used in update data from raster data to vector data. For change detection, the unchanged is mainstream, so there are lots of data which will to be updated have the same information as remote sensing data. If we excavate out the available information by computer, create remote sensing module with statistical information of the various elements, and then we can realize change detection automatically and quantitatively by information type guide, as a result to assist vector data update by human-computer interaction.

The basic idea to monitor changes in the same area of the existing historical period vector data and images is as follow. Use mature theory, for raster images such as geometric correction, image mosaic, data fusion, ect., to match vector data and image precisely together, segment the image to multiple spot unit use vector data, and pick up the spot with obvious characteristic using layering technology, and then analysis the based on the spectral characteristics of the elements of information and texture information, analyze and calculate the characteristic information of each kind of element based on spectral and texture information, and save the calculation result to files to form the characteristic information module, and then establish discrimination rules and various thresholds. After the above work, calculate and detect image characteristic information of each spot of the image, match the module information to it with discrimination rules and determine the result by the determined threshold. If the result is that the spot has changed, then we can math the characteristic information of each element in module use methods as minimum distance matching method, Bayesian (Bayes) rule, multiple matching rule, the decision tree matching rule, etc., the best matched elements is the new element class of this spot.

The technical flowchart of raster to vector data change detection as figure 1-2:

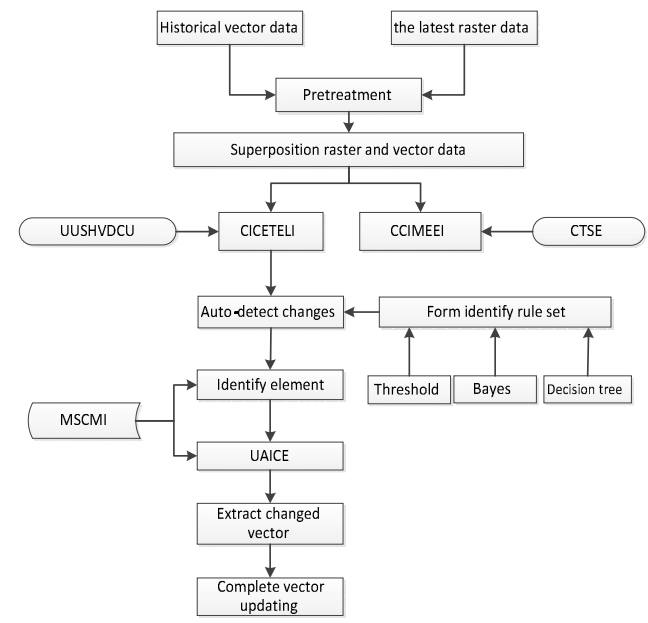

Figure 1-2 Technical flowchart of update vector data with raster data

UUSHVDCU: Use the unit spot in historical vector data as calculating unit

CICETELI: Calculate information characteristic of each type of element layering from image

CCIMEEI: Construct characteristic information module of each element from image

CTSE: Construct training sample by element 
MSCMI: Matching statistical characteristic and module information

UAICE: Update attribute information of changed element

\section{Vector to vector data update}

Vector to vector data update always occurred in topographic data and other basic geographic information data updating process, such data produced and updated framing mostly, and create database by layering element, so the database update is to utilize framing data to update the layering saved database, as a result, we can use the "version amendment" data update mode, namely "tiered storage, framing updated".

During the actual production process, local update mode is more used for vector to vector data updating, it use online editing mode and only stored the specified time status change respect to ground state.The way is to use data editing tools provided by the system, to edit each changed element directly controlled by transaction, and submit updated data to the database one-time while backup the older data. According to the collection and storage management model, frame updating or region updating will be used. By frame updating, users have to select the map sheet needs to be updated, then the system extracts data within the map sheet to local automatically from the database, after data updating, the user pass back updated data to the database and save the historical data. By region updating, data always be updated by administrative region or surveying region. System extract the region data to local according to user-selected regions, and user pass back updated data to the database after updating, at the same time, user save the historical data.

Frame updating and region updating all need to extract data within the extract scope based on updating range. When single-user offline update method used, that when a user extracts and updates a layer, other users can not extract and update the layer, the other user can only wait before the last user pass back updated data into the database. Updating process as Figure 1-3:

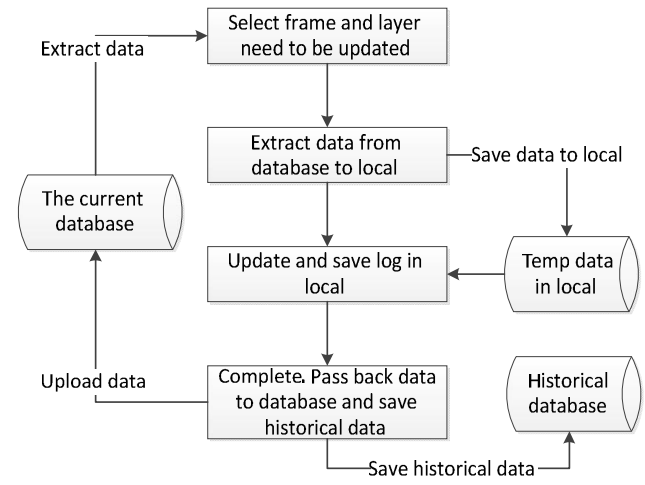

Figure 1-3 Flow chart of update vector data

\section{SUMMARIZE}

We apply these update methods to geographic information update and other production projects, the results show that these methods can improve the production efficiency effectively and also improved the accuracy of update. As in updating 1:50000 basic database, we used the raster to raster updating method(as Figure 1-4),

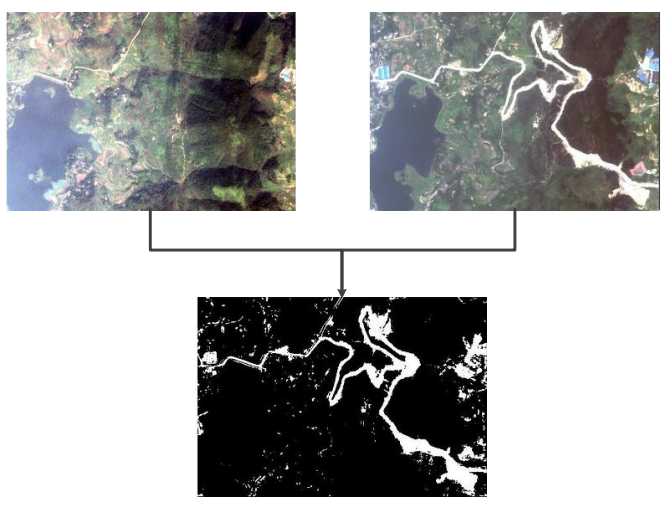

Figure 1-4 Raster to Raster Updating Application via rectified the old and new time phase images and detected the change vector, we identified the changed region effectively, it improved the efficiency of change region found and the improved the accuracy of updated data. We also used the historical vector data and the latest high-quality images to do updating, we used the raster to vector data updating method to do semi-automatic interpretation, and extracted increment between interpretation result and historical basic GIS data, and then updated the historical data based on the incremental information and specifications, the updated database is the new geographic information database. GIS data produced by this way is more 
productive, and own higher accuracy in attribute and spatial location.

\section{REFERENCES}

[1] Yuchun Pan, Ershun Zhong, Chunjiang Zhao. Spatial GIS database updating technology[J],geoinformation science, 2004,6(1):36 40.

[2] Jun Chen, Renliang Zhao, Donghua Wang, etc., Pre-test dynamic updating technology system apply

to Basic geographic information[J]. Geomatics World ,2007,10.

[3] Jun Li, Yueqin Zhou, Deren Li. Apply Wavelet transform to research fuse high-resolution panchromatic image and multispectral image[J].Journal of Remote Sensing , 1999,5.

[4] Xiulan $\mathrm{Yu}$, Guohui Qian. Layer fusion classification comparison between TM and SAR image[J].Remote Sensing Technology and Application,1999,9.

[5] Deren Li. Change monitor by using remote sensing image[J]. Journal of wuhan university (information science edition),2003,28(S1):12-17.

[6] Peijun Du, Yunhao Chen. Discussion several issues on updating geographical information by remote sensing image.[J].Science \& Technology Review , 2004(12):9-12.

[7] Zhipeng Chen. Research on difference change detection method based on texture feature[D] . Beijing: The Chinese academy of sciences institute of electronics,2003.

[8] Jun Chen, Donghua Wang, Yaoling Shang, ect., Overall design research and technology innovation on National 1:50000 database update project[J].Acta Geodaetica et Cartographica Sinica,2010,39(1):7-10.

[9] Jun Chen, Zhilin Li, Jie Jiang, ect., Continuously updated problem of basic geographic database[J].Geomatics World,2004,2(5).

[10] Xiaoguang Zhou, Jun Chen, Jianjun Zhu, ect., Incremental updating of Spatial-temporal database based on events $[\mathrm{J}]$. Journal of Image and Graphics, 2006(10): 1431 1438.

[11] Huaji Zhu. Study on incremental information classification and expression of terrain
data[D] . Beijing: Institute of remote sensing applications, Chinese academy of sciences, 2006.

[12] Qian Cheng. Research on establish the current land use database and it's updating method[D]Fuxin: Liaoning Technical University, 2005.

[13] Jinzhan Wei. Incremental updating system of spatial-temporal cadastral database design and implementation Based on Oracle Spatial[D]. Changsha: Central south university, 2005.

[14] Lu.D.S, Mausel E Batistella M, Comparison of Land-Cover Classification Methods in the Brazilian Basin $[\mathrm{J}]$, Photogrammetric Engineering and Remote Sensing. 2004:723-731. 
Author introduction: Jianping Pan (1976-), male, PHD, senior engineer, mainly engaged in remote sensing image processing and geological disaster research. E-mail: pjplj@126.com, $86+$ $23+89871827$

Funding Projects: 1. Funded by Key Laboratory of Geoinformatics of State Bureau of Surveying and Mapping,2012: Theory improving and production application research on change detection method of change vector and cross analysis; 2.scientific research and technical innovation of Chongqing Institute of Surveing and Mapping, NASMG, 2012: Research on automatically extract change vector of remote sensing image with different resolution. 